\title{
Stretching single titin molecules from failing human hearts reveals titin's role in blunting cardiac kinetic reserve
}

\author{
Mei-Pian Chen ${ }^{1,2}$, Salome A. Kiduko ${ }^{1,2}$, Nancy S. Saad ${ }^{1,2,3}$, Benjamin D. Canan ${ }^{1,2}$, \\ Ahmet Kilic $^{4}$, Peter J. Mohler ${ }^{1,2,5}$, and Paul M.L. Janssen ${ }^{1,2,5 *}$
}

\begin{abstract}
${ }^{1}$ Department of Physiology and Cell Biology, The Ohio State University, Hamilton Hall 207a, 1645 Neil Avenue, Columbus, OH 43210, USA; ${ }^{2}$ Dorothy M. Davis Heart and Lung Research Institute, 473 W 12th Ave, Columbus, OH 43210 USA; ${ }^{3}$ Department of Pharmacology and Toxicology, Faculty of Pharmacy, Helwan University, Cairo, Egypt; ${ }^{4}$ Division of Cardiothoracic Surgery, Department of Surgery, The Ohio State University Wexner Medical Center, 410 W 10th Ave, Columbus, OH 43210, USA; and ${ }^{5}$ Department of Internal Medicine, The Ohio State University Wexner Medical Center, 395 W 12th Ave, Columbus, OH 43210, USA
\end{abstract}

Received 11 September 2018; revised 8 November 2018; editorial decision 11 February 2019; accepted 13 February 2019; online publish-ahead-of-print 18 February 2019

Time for primary review: 20 days

Aims

Heart failure (HF) patients commonly experience symptoms primarily during elevated heart rates, as a result of physical activities or stress. A main determinant of diastolic passive tension, the elastic sarcomeric protein titin, has been shown to be associated with HF, with unresolved involvement regarding its role at different heart rates. To determine whether titin is playing a role in the heart rate (frequency-) dependent acceleration of relaxation (FDAR).W, we studied the FDAR responses in live human left ventricular cardiomyocytes and the corresponding titin-based passive tension (TPT) from failing and non-failing human hearts.

Methods

Using atomic force, we developed a novel single-molecule force spectroscopy approach to detect TPT based on the

and results frequency-modulated cardiac cycle. Mean TPT reduced upon an increased heart rate in non-failing human hearts, while this reduction was significantly blunted in failing human hearts. These mechanical changes in the titin distal lg domain significantly correlated with the frequency-dependent relaxation kinetics of human cardiomyocytes obtained from the corresponding hearts. Furthermore, the data suggested that the higher the TPT, the faster the cardiomyocytes relaxed, but the lower the potential of myocytes to speed up relaxation at a higher heart rate. Such poorer FDAR response was also associated with a lesser reduction or a bigger increase in TPT upon elevated heart rate.

Conclusions Our study established a novel approach in detecting dynamic heart rate relevant tension changes physiologically on native titin domains. Using this approach, the data suggested that the regulation of kinetic reserve in cardiac relaxation and its pathological changes were associated with the intensity and dynamic changes of passive tension by titin.

Keywords

Heart failure - Frequency-dependent acceleration of relaxation - Titin • Single-molecule force spectroscopy - Atomic force microscopy

\section{Introduction}

Heart failure (HF) remains the leading cause of death, and more than half of the patients with HF suffer from both systolic and diastolic dysfunctions. ${ }^{1,2}$ Humans rely highly on a healthy heart that not only beats stronger, but also contracts and relaxes faster upon an increased demand of cardiac output. ${ }^{3}$ This property of cardiac relaxation, known as frequency-dependent acceleration of relaxation (FDAR), is impaired in failing human myocardium., ${ }^{4,5}$ Despite potential mechanisms suggested, the molecular machinery behind pathological FDAR remains elusive. ${ }^{6-8}$ Manifestations include prolonged relaxation, smaller enddiastolic volumes, higher end-diastolic left ventricular (LV) stiffness, and elevated diastolic force in myocardium. ${ }^{4,5}$, The kinetics of intracellular calcium decline are accelerated as frequency increases, while myofilament calcium sensitivity decreases, ${ }^{7}$ though the decline of calcium transient does not directly reflect relaxation. ${ }^{1}$ FDAR occurs at single cell level, ${ }^{7}$ having different mechanical properties in human than other animals. ${ }^{3}$ Studies of FDAR and its abnormalities on human cardiomyocytes are crucial to better understand diastolic dysfunction, but such data are currently lacking.

* Corresponding author. Tel: 614247 7838; fax: (614) 292-4888, E-mail: janssen.10@osu.edu

Published on behalf of the European Society of Cardiology. All rights reserved. (c) The Author(s) 2019. For permissions, please email: journals.permissions@oup.com. 
The rate of myocardial relaxation, particularly in the later phases, is suggested to be regulated predominantly by myofilaments. ${ }^{10-12}$ Titin, the giant myofilament protein aligns through the sarcomere successively, and determines myocardium passive tension during diastole. ${ }^{13,14}$ Titin is also crucial for myocardial stiffness, myofibril assembly, ${ }^{15}$ and contractile function. ${ }^{16}$ Isoform switching, phosphorylations, degradation, and mutations of titin have been associated with various cardiac diseases. ${ }^{15,17,18}$ Recent studies show significant increases of titin-based passive tension (TPT) in patients with hypertrophic or dilated cardiomyopathy ${ }^{19}$ and HF patients with hypertension and preserved ejection fraction. ${ }^{20}$ However, the mechanisms behind the common phenomenon that patients experience symptoms only during physical activities or stress, but not at rest ${ }^{5,21,22}$ remain unresolved.

To identify the role of titin in HF diastolic kinetics, we developed a novel approach using atomic force microscopy (AFM) to detect TPT by tethering the elastic domains of single titin molecules isolated from failing and non-failing human hearts. AFM allows direct measurement of the physical properties of single molecules at force and length resolution on the order of piconewtons and nanometres, respectively. AFM force spectroscopy has been widely used on analysing different proteins, with titin as the best-known molecule studied. ${ }^{23}$ TPT was measured physiologically based on dynamic ventricular volume changes in the normal cardiac volume cycle. From the corresponding human hearts in which TPT was measured, live cardiomyocytes were freshly isolated for assessment of cell shortening at different frequency stimulations. We showed that positive FDAR occurred in myocytes isolated from non-failing human hearts, but this response was blunted in the myocytes from end-stage failing hearts. FDAR was correlated with the changes in TPT at higher heart rates, which was diminished in the failing hearts. Using our novel approach in measuring human native titin mechanics physiologically, our results provided not only cellular mechanical evidence but also molecular insights into impaired myocardial relaxation of human HF.

\section{Methods}

\subsection{Human hearts processing}

Failing human hearts were obtained from patients with end-stage HF undergoing cardiac transplantation at The Ohio State University Wexner Medical Center. Written informed consents were acquired beforehand as approved by the Institutional Review Board approval of The Ohio State University (IRB 2008H0113 and IRB 2012H0197), in accordance with the 1964 Declaration of Helsinki and its later amendments. Nonfailing donor hearts were obtained from the Lifeline of Ohio Organ Procurement programme (https://lifelineofohio.org). The Institutional Review Board had waived the need of consent for these tissues, which were used according to the Ohio State University guidelines regarding the use of data and/or specimens from diseased individuals. The characteristics of these hearts are provided in Table 1.

Upon explantation, the hearts were immediately (within seconds) perfused through the coronary arteries with ice-cold cardioplegic solution containing (in mM) $110 \mathrm{NaCl}, 16 \mathrm{KCl}, 16 \mathrm{MgCl}_{2}, 10 \mathrm{NaHCO}_{3}, 0.5$ $\mathrm{CaCl}_{2}, \mathrm{pH} 7.4$, then weighed and processed within 30 min after excision. A wedge from LV lateral mid-wall was dissected for cardiomyocyte isolation. Adjacent tissues were collected in small pieces, snap frozen in liquid nitrogen and stored in $-80^{\circ} \mathrm{C}$ for titin mechanics studies.

\subsection{Human cardiomyocytes isolation}

Live myocytes were freshly isolated from the wedge dissected from the human heart LV lateral mid-wall by enzymatic perfusions. Details are given in the Supplementary Methods section. Rod-shaped myocytes with clear striation and edges were selected for contractility tests, which were conducted within $8 \mathrm{~h}$ of isolation.

\subsection{Cardiomyocyte contractility}

Isolated human cardiomyocytes were settled in a microscope chamber (Cell MicroControls, Norfolk, VA) for 2 min with incubation buffer perfused at body temperature $\left(37^{\circ} \mathrm{C}\right)$, followed by acclimatization for at least $15 \mathrm{~min}$ by field stimulation at $0.5 \mathrm{~Hz}$ using a pair of platinum wires using a bipolar pulse (Cell MicroControl). Cells were then stimulated at $0.5,1.0,1.5 \mathrm{~Hz}$ consecutively with at least $2 \mathrm{~min}$ of stimulation for each frequency recording which allowed contraction to reach steady state. Sarcomere shortening was continuously recorded on the video-based myocyte contractility system (lonOptix LLC, Westwood, MA). Data were analysed based on the averages of at least 10 representative contractions from each condition. Results from three or more cardiomyocytes were averaged for each human heart, and treated as $n=1$ ( 5 nonfailing, 11 failing hearts), not all myocytes could be stimulated to contract at all frequencies. Parameters in myocyte relaxation, including velocity of relaxation over peak height ( $\mathrm{Vrel} /$ peak h), time from peak tension to $50 \%$ and $90 \%$ relaxation (RT50 and RT90) were analysed.

\subsection{Preparation of protein}

Protein was extracted from frozen LV lateral mid-wall tissue from failing $(n=11)$ and non-failing $(n=7)$ human hearts. Details are in Supplementary Methods section.

\section{5 Force spectroscopy for specific tethering identification}

Single-molecule force spectroscopy (SMFS) of the titin PEVK-distal Ig domain and the Ig domain alone was performed on an automated atomic force microscope (ForceRobot ${ }^{\circledR} 300$, JPK Instruments, Berlin, Germany) by tethering between two pairs of antibodies, 9D10 ${ }^{24}$ and $\mathrm{T} 11^{25}$ for PEVK [rich in proline $(P)$, glutamate I, valine $(V)$ and lysine $(K)$ ]-distal immunoglobulin (Ig) domain, $184^{26}$ and T11 for Ig domain alone. Antibodies used here had been tested for their specificity previously and had long been used in titin immunoblotting, immunofluorescence microscopy, and immunoelectron microscopy. 9D10 labels PEVK domain, previously used in SMFS for native skeletal muscle titin. ${ }^{27,28} \mathrm{~T} 11$ stains the region of A-I junction in the sarcomere, labelling the C-terminal end of distal Ig domain. ${ }^{25} 184$ labels the region where PEVK and distal Ig domain connects. ${ }^{29-32}$ Preparation details are provided in Supplementary Methods section. For specific tethering identification, loadings of antibodies 9D10 or 184 were eliminated for the control without antibody on the probes, and were additionally added to the testing buffer prior to SMFS for the control blocking the respective antigen binding sites.

Protein extract from one of the failing human hearts was used in the experiment. AFM probes approached to the glass surface and retracted at $1.5 \mu \mathrm{m} / \mathrm{s}$ for $1 \mu \mathrm{m}$, with probe-glass contact at $0.5 \mathrm{nN}$ for $0.5 \mathrm{~s}$, ${ }^{33,34}$ allowing antibody-antigen reaction. Data of $1000 \pm 300$ approaches were collected for antibody 9D10 and $3000 \pm 700$ approaches for 184 . Using the manufacturer's data processing software (JPKSPM 5.1.8), force vs. distance relationship at protein-probe detachment, where unfolding force dropped sharply to baseline, was analysed and overlaid. Quadrant analysis was drawn and probabilities of events in each quadrant were calculated as the data counts in each quadrant over the total events in each experiment. Three independent experiments were repeated for each experimental condition. 
Table I Donor and patient demographics

\begin{tabular}{|c|c|c|c|c|c|c|c|c|}
\hline Heart number & Age & Gender & Race & EF (\%) & Cause of death & Etiology & NYHA class (1-4) & ACC stage (A-D) \\
\hline \multicolumn{9}{|l|}{ Non-failing hearts } \\
\hline 240603 & 51 & $\mathrm{~F}$ & Caucasian & $40-50$ & Blunt head trauma, non-MVA & & & \\
\hline 331253 & 42 & $\mathrm{~F}$ & Hispanic & 55 & $\mathrm{CVA}, \mathrm{ICH}$ & & & \\
\hline 435578 & 20 & M & Caucasian & 35 & Drug overdose, anoxia & & & \\
\hline 452192 & 55 & $\mathrm{~F}$ & African-American & 60 & CVA, stroke & & & \\
\hline 460025 & 69 & $\mathrm{~F}$ & Caucasian & 60 & $\mathrm{CVA}, \mathrm{ICH}$ & & & \\
\hline 958987 & 40 & M & Caucasian & 60 & Drug overdose, cardiac arrest & & & \\
\hline 987692 & 34 & $\mathrm{~F}$ & Caucasian & 30 & MVA, trauma & & & \\
\hline \multicolumn{9}{|l|}{ Failing hearts } \\
\hline 280147 & 63 & $\mathrm{~F}$ & African-American & 40 & & NICM & 3 & $D$ \\
\hline 335581 & 40 & $\mathrm{~F}$ & Caucasian & $50-55$ & & NICM & 3 & C \\
\hline 369452 & 61 & M & African-American & $15-20$ & & NICM & 3 & $D$ \\
\hline 390112 & 60 & $\mathrm{~F}$ & Caucasian & $<20$ & & NICM & 3 & $D$ \\
\hline 554147 & 56 & M & Caucasian & $<20$ & & NICM & 1 & $D$ \\
\hline 569897 & 48 & M & Caucasian & $20-25$ & & NICM & 1 & $D$ \\
\hline 631231 & 49 & $\mathrm{~F}$ & Asian & $20-25$ & & $\mathrm{CICM}$ & 3 & $D$ \\
\hline 645444 & 47 & M & African-American & $<20$ & & ICM & 3 & $D$ \\
\hline 777902 & 59 & M & Caucasian & 20 & & CAD & 3 & $D$ \\
\hline 963542 & 60 & $\mathrm{~F}$ & Caucasian & 20 & & NICM & 3 & $D$ \\
\hline 994744 & 53 & M & Caucasian & $10-15$ & & NICM & 4 & $D$ \\
\hline
\end{tabular}

ACC, American Heart Association; CAD, coronary artery disease; CICM, chemotherapy induced cardiomyopathy; CVA, cerebrovascular accident; EF, ejection fraction; F, female; ICH, intracerebral haemorrhage; ICM, ischaemic cardiomyopathy; M, male; MVA, motor vehicle accident; NICM, non-ischaemic cardiomyopathy; NYHA, New York Heart Association Class.

Table 2 Force spectroscopy setting for titin mechanics test based on cardiac cycle by AFM

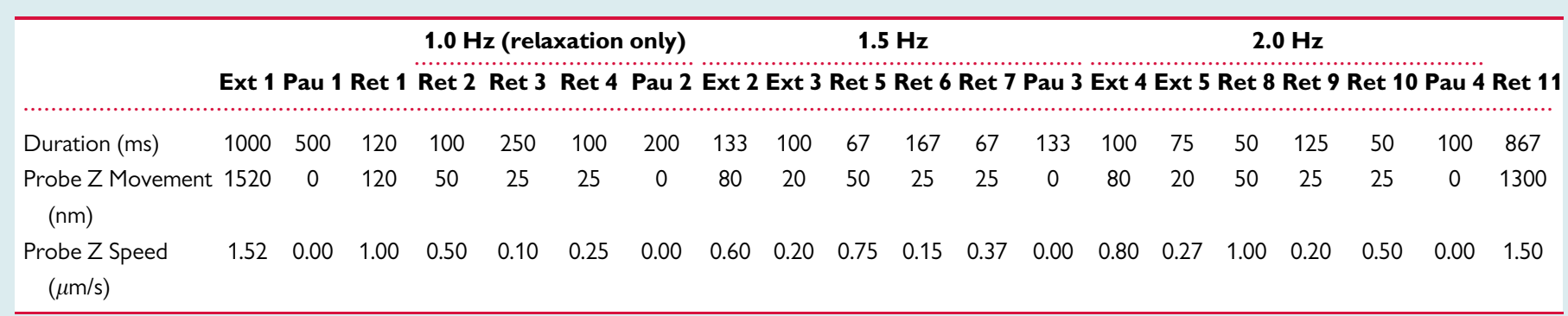

Ext, extend; Pau, pause; Ret, retract.

\subsection{SMFS at cardiac cycle}

Following the same preparation of the SMFS experiment for specific tethering identification, samples from the same hearts with myocyte contractility tested and two additional non-failing hearts (total 7 non-failing, and 11 failing hearts) were measured for SMFS based on ventricular volume changes of cardiac cycle. The AFM contact mode protocol was set based on cardiac LV volume changes as they would occur during an in vivo ejecting beat (i.e. like a Wiggers volume diagram), as shown in Table 2, having the curve of ventricular volume changes approximated by a series of continuous straight lines. Details are in Supplementary Methods section.

\subsection{Statistics}

Pearson analysis was used to study correlations. Mixed-effects analysis with Tukey-Kramer multiple comparisons test was applied on comparisons among data from different frequency stimulations on cardiomyocytes.
Kruskal-Wallis test with Conover-Iman (multiple comparisons was used for probability analysis on titin). Unpaired $t$-test was used to compare data between groups of failing and non-failing hearts. Paired $t$-test was used to compare data of relative TPT measured at two different heart rates within the same titin domain. A $P<0.05$ was considered statistically significant. All data was shown as mean \pm standard error of mean (SEM).

\section{Results}

\subsection{FDAR occurred in isolated human cardiomyocytes but was blunted in failing hearts}

Isolated viable rod-shaped cardiomyocytes showed clear striation and edges. The yield was typically within $30-50 \%$ by the final cell suspension 
A

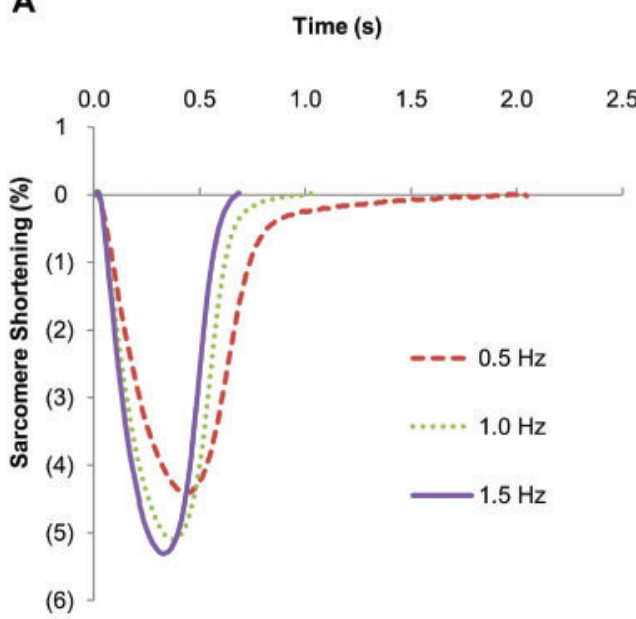

B
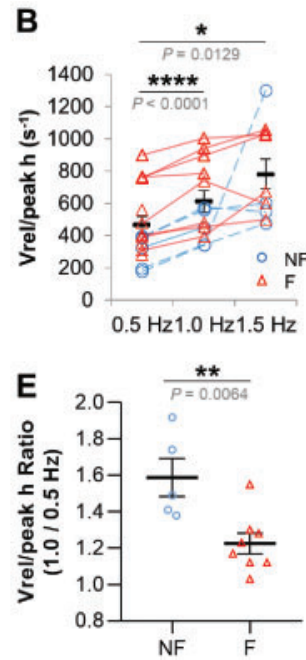

C
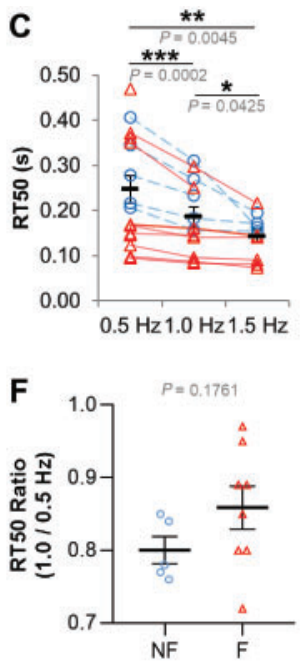
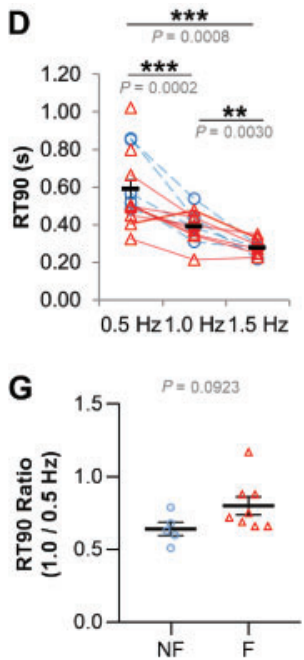

Figure I Frequency-dependent relaxation kinetics of human cardiomyocytes. (A) Representative sarcomere shortening traces obtained in a cardiomyocyte isolated from a non-failing human heart. (B-D) Average Vrel/peak h, RT50 and RT90 of myocytes isolated from both non-failing $(n=5)$ and failing $(n=11)$ human hearts (NF: $n=5,5$, and 4, and F: $n=11,8$, and 6 for $0.5,1$, and $2 \mathrm{~Hz}$, respectively). Analysed by mixed-effects analysis with Tukey multiple comparisons. $P=0.2059$ for $1.0 \mathrm{~Hz}$ vs. $1.5 \mathrm{~Hz}$ in $(B)$. $(E-G)$ Comparisons of the rates in the acceleration of relaxation in the myocytes isolated from failing $(F)$ and non-failing (NF) human hearts. Analysed by unpaired $t$-test. Data showed as mean $\pm \mathrm{SEM}$.

in a successful cell isolation. No significant difference was observed in the morphology of myocytes isolated from the failing and non-failing hearts. With the increasing frequency of stimulations, myocytes (combining data from both the failing and non-failing hearts) sped up relaxation significantly (Figure $1 A$ ), indicated by the increasing velocity of relaxation (Figure $1 B$ ), and the decreasing time to relax (Figure $1 C$ and D). By comparing the mean ratios at 1.0 over $0.5 \mathrm{~Hz}$, myocytes from the failing hearts showed blunted FDAR, as indicated by the lesser increase in Vrel/ peak $h$, and the lesser reduction in RT50 and RT90 (Figure 1E-G). Similarly, the mean ratios of $\mathrm{Vrel} /$ peak $h$ at 1.5 over $1.0 \mathrm{~Hz}$ were $1.81 \pm 0.68$ in non-failing group while $1.15 \pm 0.12$ in failing group. These ratios were $0.79 \pm 0.11$ and $0.90 \pm 0.04$ for $R T 50,0.66 \pm 0.09$ and $0.80 \pm 0.06$ for RT90 in non-failing and failing groups, respectively.

\subsection{Identification of antibody specific tethering of titin elastic domains}

From the same human hearts we used to access FDAR, we measured and compare mechanical properties of single titin molecule using AFM. The figure represents the analysis of all the AFM attempts on a single heart, and was performed on each heart (not shown). During cardiac relaxation, titin is unfolded firstly at tandem Ig domain, followed by PEVK segment, and then N2 domains. ${ }^{35-37}$ We tested SMFS on native titin PEVK-distal Ig domain and distal Ig domain alone using domain specific antibodies (Figure 2A). ${ }^{38-40}$ Experimental controls were applied to identify specific tethering (Figure $2 B$ ). The AFM probe picked up and unfolded molecules on retractions (Figure $2 C$ ), at the end of which unfolding force dropped sharply to baseline on protein-probe detachments. By the quadrant analysis of the force vs. distance relationship at protein-probe detachment from the three experiment conditions overlaid (Figure 2D and $E$, left), specific tethering events were identified at the upper right quadrants. These quadrants contained the biggest probability differences among the groups (Figure 2D and E, right). This quadrant of antibody specific tethering for PEVK-distal Ig domain was set at protein-probe detached at over $1.3 \mathrm{nN}$ in force and over $300 \mathrm{~nm}$ in probe-sample separation; while the quadrant for tethering distal Ig domain alone was set at over $1.0 \mathrm{nN}$ and $200 \mathrm{~nm}$ at protein-probe detachment. The identification of antibody specific tethering that detached with high force and long stretching distance was consistent with the tight binding property between antibodies and protein antigens. ${ }^{41}$

\subsection{Titin SMFS based on cardiac cycle}

To measure titin mechanics more physiologically by AFM, we established a SMFS protocol based on the dynamic ventricular volume changes of cardiac cycle as illustrated in the Wiggers diagram ${ }^{42}$ (Figure $3 A$ ). Titin domains were stretched as the way the heart relaxes at $1.0 \mathrm{~Hz}$, followed by full contraction-relaxation cycles at 1.5 and $2.0 \mathrm{~Hz}$. A last step with a full unfolding of the domains was included to identify antibody specific tethering as aforementioned.

Interestingly, from the series of linear stretch and release protocol, the force tracings of the two titin domains showed a nonlinear relationship with time, reconstituting a similar pattern as the curve of ventricular volume changes in the Wiggers diagram (Figure 3B). Such phenomenon reflected the viscoelasticity of the two titin domains, and it shared among those specific tethering events, especially when tethering at 1.5 and $2.0 \mathrm{~Hz}$. Our result showed the SMFS approach here was able to reveal the mechanical properties of the titin PEVK-distal Ig domain and the distal Ig domain in vitro in the manner that is close to the way they prevail in the sarcomere in vivo.

\subsection{Mean TPT reduced at faster cardiac cycle but at a lesser extent in failing hearts}

TPT was assessed from the same hearts the myocyte shortening was tested (with two additional non-failing hearts). TPT was measured as the force differences between the lowest and the highest force detected 

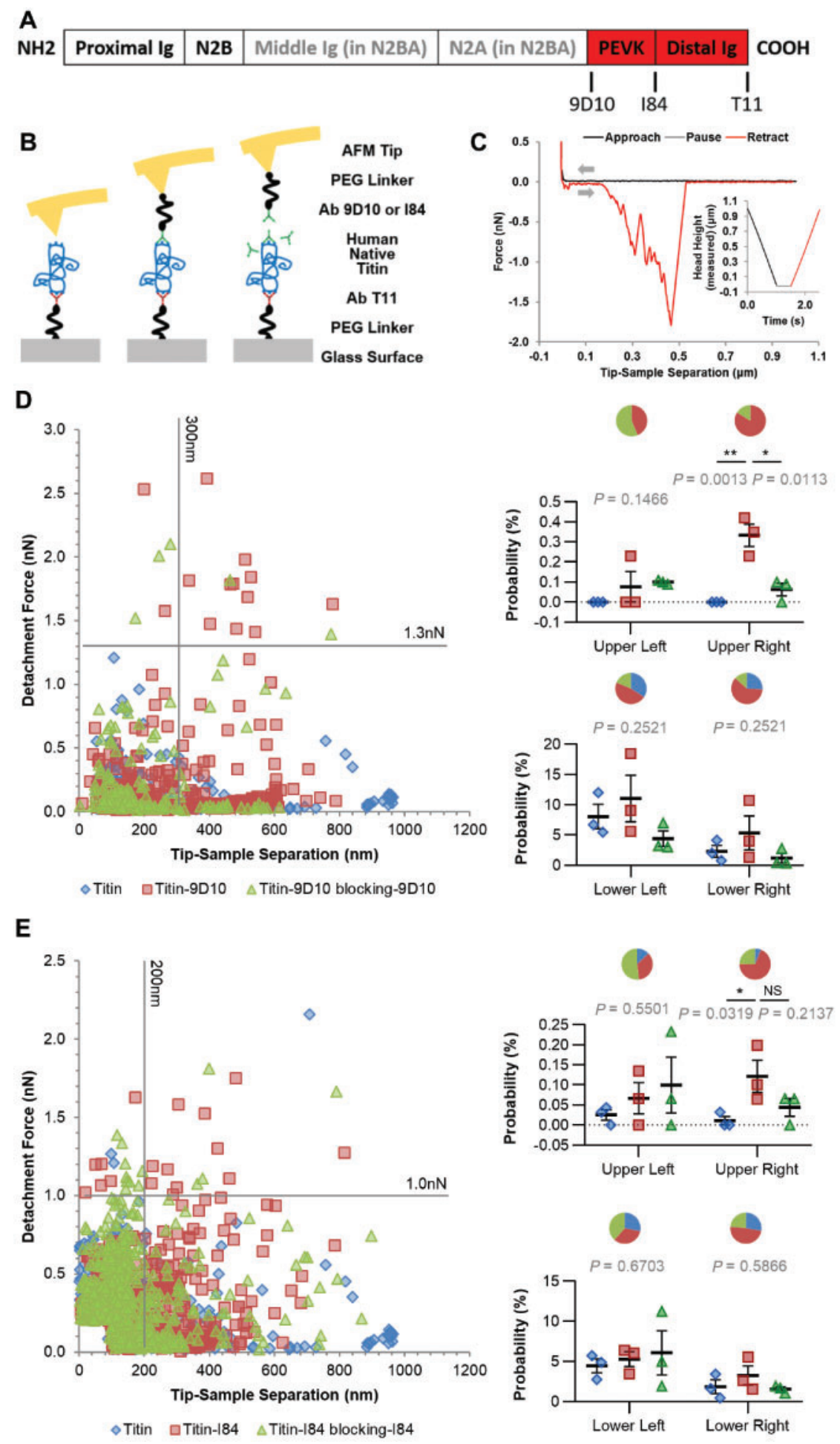

Figure 2 Identification of antibody specific tethering of titin domains from human hearts, displays as all AFM attempts on a single heart, and was repeated for all hearts. (A) Domain structure of I-band segment of human cardiac titin and antibody binding sites of 9D10, 184, and T11. PEVK and distal Ig domain tested were highlighted in red. Regions presented in titin N2BA but not in N2B isoform were labelled in grey. (B) Schematic illustration of experimental design for detection of titin PEVK-distal Ig domain and Ig domain alone specific tethering. Conditions of specific tethering (middle), without antibody on the probe (left) and with excessive antibody blocking (right) are showing. (C) Representative force-extension traces of titin PEVK-distal Ig domain specific tethering. Inset shows the force spectroscopy protocol. (D, E) Left panels, Typical examples of overlaid scatter plots of force vs. distance relationships at proteinprobe detachment obtained from the three indicated experimental conditions for PEVK-distal Ig domain (D) and Ig domain alone (E); right panels, probabilities of each experimental condition appears in the quadrants drawn in the scatter plots. Statistical analysis performed with Kruskal-Wallis test with Conover-Iman multiple comparisons. 

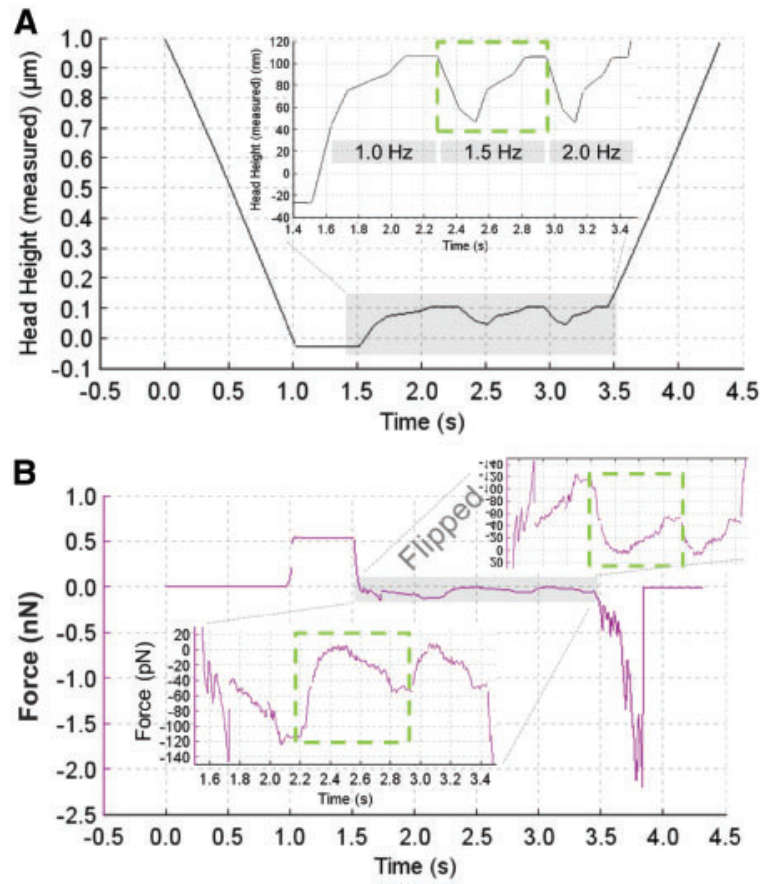

Figure 3 Force spectroscopy of titin domains from human hearts based on ventricular volume changes of cardiac cycle. (A) AFM probe height vs. time relationship of the SMFS protocol. The inset is an enlargement of the grey region on the protocol setting. (B) Force vs. time relationship of a representative SMFS tracing of titin PEVK-distal Ig domain. The lower inset is an enlargement of the grey region; the upper inset is an up-down flipped figure of the lower inset, converting the force increase upwards, and showing a similar pattern as the ventricular volume changes in Wiggers diagram.

Table 3 Comparison of TPT Ratio at 2.0/1.5 Hz obtained from the regular protocol and the reverse protocol of SMFS at cardiac cycle

\begin{tabular}{|c|c|c|}
\hline Heart number & From 1.5 to $2.0 \mathrm{~Hz}$ & From 2.0 to $1.5 \mathrm{~Hz}$ \\
\hline 240603 & 0.95 & 0.95 \\
\hline 335581 & 0.93 & 0.97 \\
\hline 569897 & 1.09 & 1.08 \\
\hline 994744 & 1.17 & 1.35 \\
\hline
\end{tabular}

when stretching titin domains at 1.5 and $2.0 \mathrm{~Hz}$ (Figure 4A). The mean value of TPT detected in each sample ranged from 21.2 to $265.2 \mathrm{pN}$. Relative TPT was analysed, which was the ratio of TPT to the corresponding force when protein-probe detached in each tethering event, excluding potential differences in antibody-antigen binding including cases with two or more molecule binding events.

Combining data from both failing and non-failing human heart samples, the mean relative TPT in both PEVK-distal Ig domain and distal Ig domain alone was significantly reduced from 1.5 to $2.0 \mathrm{~Hz}$ (Figure 4B). The results were consistent in both the titin domains, despite the insignificant correlation in the relative TPT from the two titin domains (data not shown). This frequency-dependent change of TPT was independent of the frequency setting in the protocol, as indicated by the consistent result from the reverse protocol stretching titin domains from 2.0 to $1.5 \mathrm{~Hz}$ (Figure 4A, Table 3).

The mean relative TPT in distal Ig domain alone of non-failing hearts was significantly reduced from 0.054 to $0.050(P=0.0011)$ tethering from 2.0 to $1.5 \mathrm{~Hz}$, with the ratio of 0.91 at 2.0 over $1.5 \mathrm{~Hz}$. However, this reduction of TPT at faster cardiac cycle was significantly blunted in failing hearts, with the 2.0 over $1.5 \mathrm{~Hz}$ ratio of 0.99 (Figure 4 C). Similar trend existed in PEVK-distal Ig domain, with a positive correlation to the TPT changes in distal lg domain alone (Figure 4D).

\subsection{TPT correlated to the relaxation kinetics of cardiomyocytes}

To identify the role of TPT in frequency-dependent myocardial relaxation, we next studied the correlation between the relative TPT measured in AFM and the relaxation kinetics accessed from myocytes isolated from the corresponding human hearts. TPT of distal Ig domain measured both at 1.5 and $2.0 \mathrm{~Hz}$ was correlated positively with myocyte $\mathrm{Vrel} /$ peak h (Figure 5A), and negatively with RT50 (Figure 5B). Thus, the higher the TPT, the faster the cardiomyocytes relaxed. However, the higher the TPT, the lower the potential of myocytes to speed up relaxation at a higher heart rate, as indicated by the positive correlation between relative TPT of distal Ig domain at $2.0 \mathrm{~Hz}$ and $\mathrm{RT50}$, RT90 ratios of myocytes at $1.0 / 0.5 \mathrm{~Hz}$ (Figure $6 \mathrm{~A}$ and $\mathrm{B}$ ). Moreover, lesser the TPT reduction or bigger the TPT increase at higher frequency was associated with a poorer FDAR (Figure 6C). Although correlation studies here combined data from failing and non-failing heart samples, trends existed with failing hearts that had lesser TPT reduction or even increases at higher heart rate in conjunction with poorer FDAR.

\section{Discussion}

In this study, we showed that passive tension on native titin domains could be assessed by stretching titin molecules at physiologically relevant dynamics, closely following those titin would undergo in the beating heart in vivo. We found TPT decreased averagely at higher heart rates, but at a lesser extent in failing hearts. The results were significantly correlated with the relaxation property of the contracting myocytes isolated from the corresponding human hearts.

\subsection{Consideration for studying cardiac kinetics using human heart samples}

Mechanical properties of the heart in big animals are critically different from that in small animals, in terms of the speed, cardiac reserve, and regulation of these parameters. For instance, cardiac output in mice can be maximally doubled during exercise (typically actually only $\sim 20-40 \%$ ), while in human it can be increased as much as by 5 - to 10 -fold. ${ }^{3}$ Moreover, titin-based viscosity, which depends on the speed that titin slides over other sarcomeric proteins, is 5-10 times lower in human comparing to small animals. ${ }^{43}$ In addition, the variety in the human patient population allows for correlation studies, since the variability in the results is genomic/phenotype-based, instead of experimental error/ variability-based. Additionally, the strength and statistical significance of association found in heterogeneous populations is considered to be stronger. 

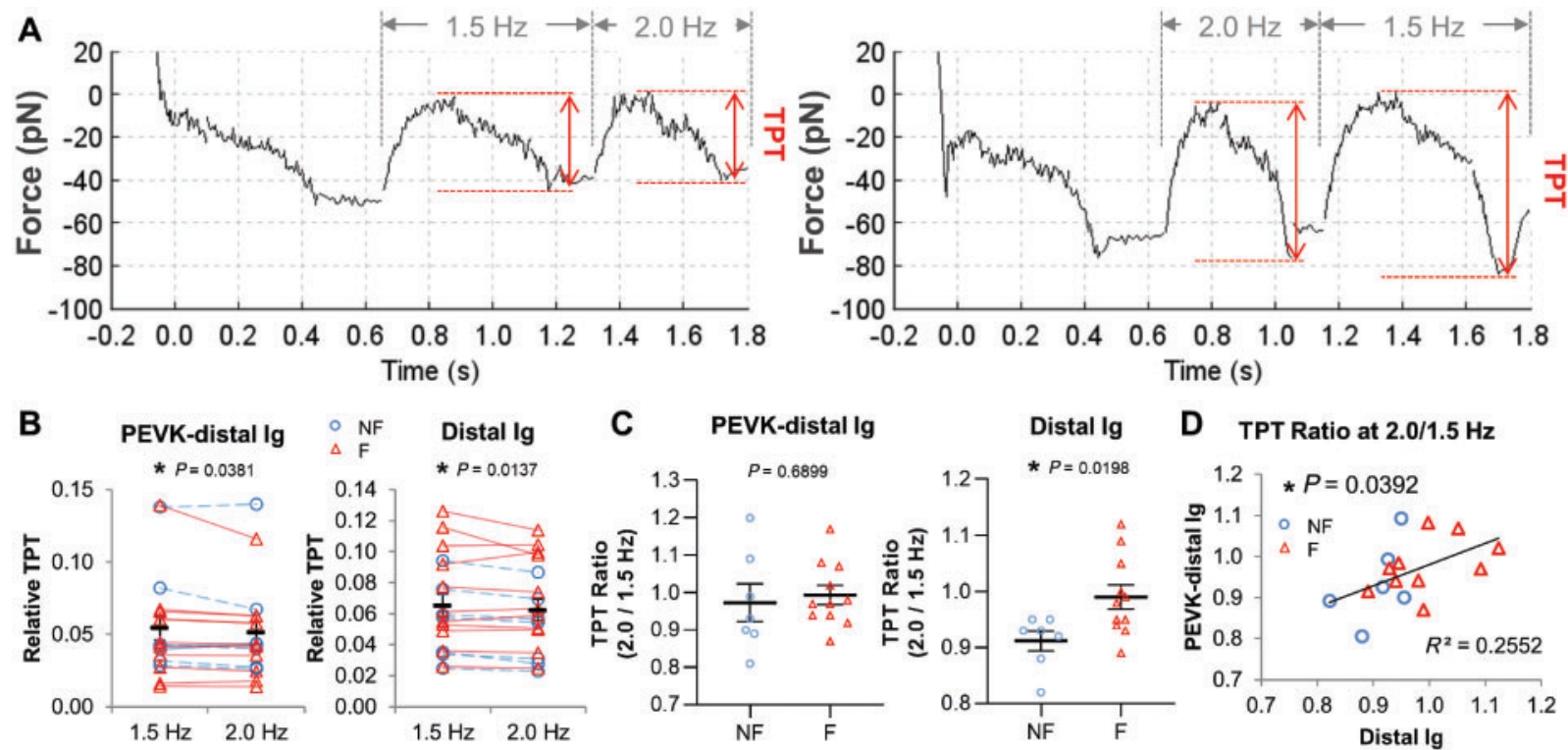

D TPT Ratio at 2.0/1.5 Hz

Figure 4 Tension differences in titin domains at different heart rates in failing and non-failing human hearts. (A) Representative SMFS tracings of titin distal Ig domain from a non-failing human heart at cardiac cycle tethering from 1.0 to 1.5 then $2.0 \mathrm{~Hz}$ (left) and from 1.0 to 2.0 then $1.5 \mathrm{~Hz}$ (right) obtained from the same experiment. Labels in red, the measurement of TPT, which is the force differences between the lowest and the highest force detected at each cardiac cycle. (B) Mean relative TPT of all the tested human samples $(n=18)$ at 1.5 and $2.0 \mathrm{~Hz}$. Significant reductions on the mean relative TPT were shown at faster heart rate. $* P<0.05$ by paired $t$-test. (C) Mean TPT ratios of failing and non-failing human hearts at 2.0 over $1.5 \mathrm{~Hz}$. The TPT reductions at higher heart rate were blunted in failing human hearts, with significant difference in distal lg domain. * $P<0.05$ by unpaired $t$-test. (D) TPT ratios $(2.0 / 1.5 \mathrm{~Hz})$ positively correlated between PEVK-distal Ig domain and distal Ig domain in combined human data. $* P<0.05$ by Pearson analysis.

\subsection{Innovation in native titin SMFS}

Titin SMFS has been established since 1997 with AFM $^{44}$ and with optical tweezers. $^{8,45}$ While most studies use recombinant fragments, ${ }^{33,34,46-50}$ native animal titin has been isolated for SMFS, $8,27,44,45,51$, with dominant degradation products shown from the multiple centrifugation process. ${ }^{51}$ However, manipulation in its preparation process must to be minimized to identify potential differences in titin mechanics between health and disease.

Antibody labelling has been used in SMFS with isolated native animal titin. ${ }^{27}$ Taking the advantage of the previous approach on identifying antibody-antigen interaction in AFM, ${ }^{41}$ we developed a novel SMFS approach to identify specific tethering of the native titin domains by holding between two titin antibodies. By eliminating the titin isolation process, measurements increased the sensitivity in differentiating pathological changes of titin mechanics. Moreover, comparing to SMFS with isolated titin, our approach preserved possible interactions between titin domains and other molecules, which might contribute to TPT in vivo.

Specific tethering events were revealed by data overlaying with two control experiments, two data parameters, and quadrant analysis. Similar results in antibody specificity identification were found in both the titin domains, albeit using different antibodies. Furthermore, shorter length found in protein-probe detachment agreed with the smaller domain length in Ig domain alone.

\subsection{Detecting titin mechanics physiologically}

Previous titin SMFS approaches have been focusing on full unfolding or stretching to the length well exceeding the physiological upper limit that can be reached in the sarcomere. However, large-scale Ig domain unfolding has been identified to be physiologically irrelevant, instead, only 3\% of the modules considered to be unfolded depending on stretch amplitude. $^{35,52-54}$ The same notion can be extrapolated to the PEVK domain, which extends after Ig domains. ${ }^{35-37}$ Therefore, accessing titin SMFS within or closer to its normal physiological limits is essential to identify its potential role in HF.

To maximize translation of the results, we developed a titin SMFS protocol based on the ventricular volume changes of a cardiac cycle as classically illustrated in the Wiggers diagram. ${ }^{42}$ In this protocol, titin elastic domains were stretched proportionally in the way they undergo with the ventricular volume changes during a cardiac cycle. The force tracings reconstituted a similar pattern as the curve of ventricular volume changes in Wiggers diagram, suggested dynamic non-linear force changes contributing to the cardiac cycle by the titin domains. The non-linear force changes here agreed with the well-known titin viscoelasticity, which had been suggested to arise mainly from the Ig domain and some from PEVK domain according to previous Monte Carlo simulations. ${ }^{54}$ Each tethering event included the last step to identify the specificity that held between the two antibodies. The rupture force at the end for specificity identification was not affected by the previous steps of cardiac cycles, with its reading at around 10 -fold higher.

\subsection{Role of titin in cardiac kinetic reserve}

Recent studies on cardiac TPT of patient ${ }^{19,20}$ or animal ${ }^{13}$ are based on the developed test using skinned myocardial fibres. Nevertheless, with its limitations in studying frequency-dependent titin mechanics physiologically to explain the common phenomenon that HF patients 

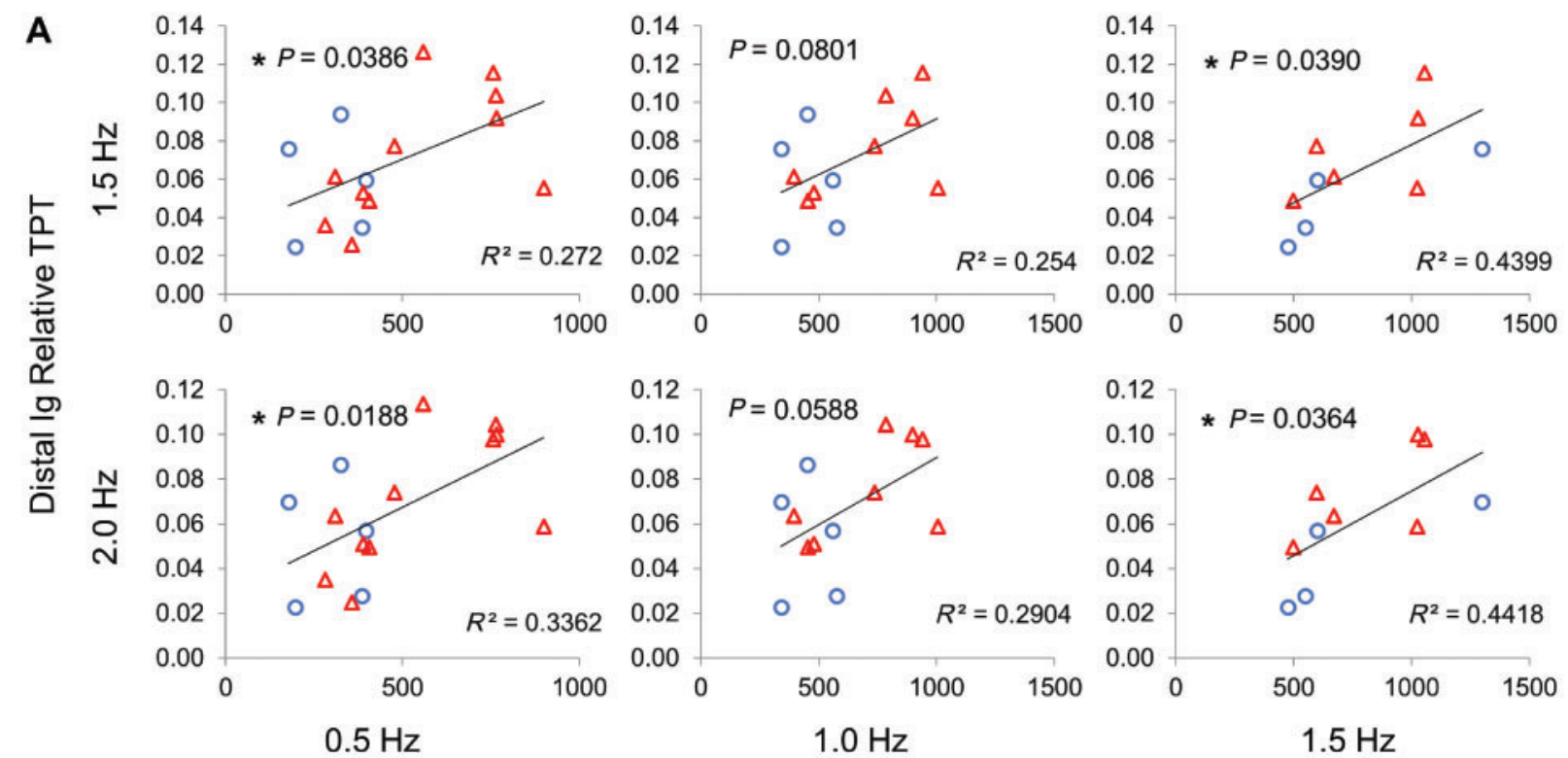

\section{Cardiomyocytes Vrel/peak $\mathrm{h}\left(\mathrm{s}^{-1}\right)$}
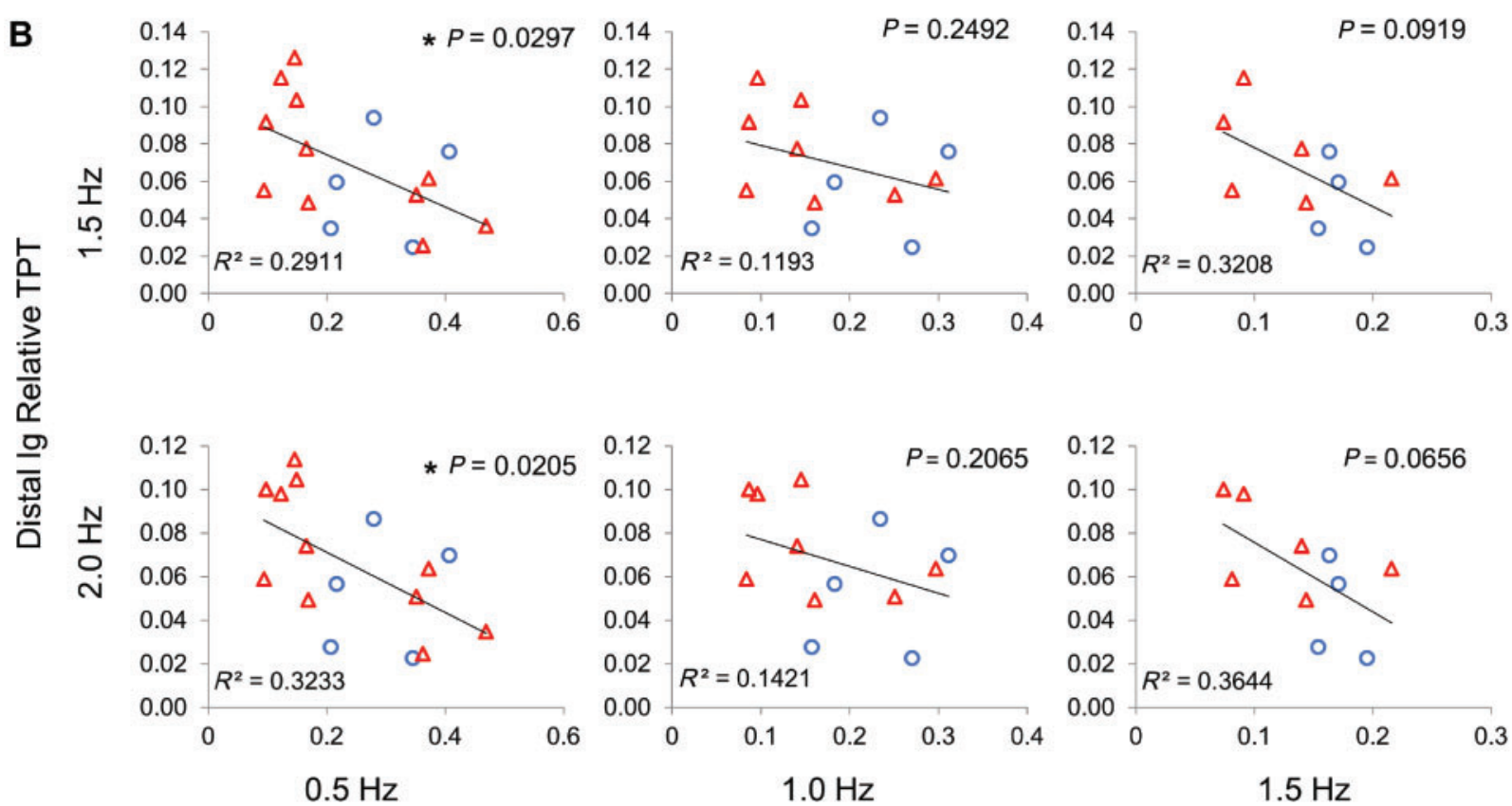

\section{Cardiomyocytes RT50 (s)}

Figure 5 Relationship between tension by titin distal lg domain and relaxation kinetics of cardiomyocytes in human hearts. Relative TPT of distal lg domain at 1.5 and $2.0 \mathrm{~Hz}$ was positively correlated with $\mathrm{Vrel} /$ peak $\mathrm{h}(\mathrm{A})$ and negatively correlated with $\mathrm{RT} 50(B)$ of isolated cardiomyocytes paced at $0.5,1.0,2.0 \mathrm{~Hz}$ (NF: $n=5,5$, and 4, and F: $n=11,8$, and 6, respectively). The correlations suggested that the higher the TPT, the faster the myocytes relaxed. Statistic was done by Pearson analysis, $* P<0.05$.

experience symptoms primarily during physical activities or stress, we developed the current approach for native titin SMFS. The force differences between the lowest and the highest force measured at cardiac cycle here, was to measure TPT from the end systolic volume to the enddiastolic volume of the heart.
Although end-stage HF is often classified into systolic or diastolic HF, both types of dysfunctions occur simultaneously, with the possibility of one being dominant over another. ${ }^{55}$ Both systolic and diastolic HF are characterized by a decrease in exercise tolerance, ${ }^{56}$ which has also been shown in titin proximal Ig domain knockout mice. ${ }^{26}$ To further elucidate 
A

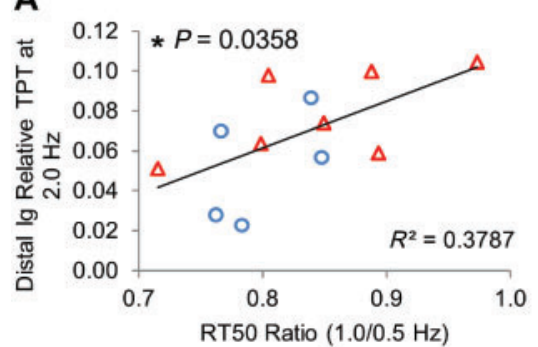

B

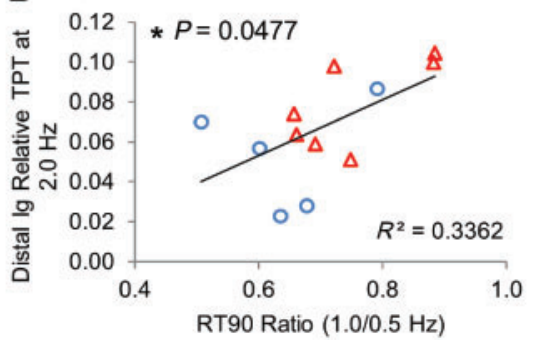

C

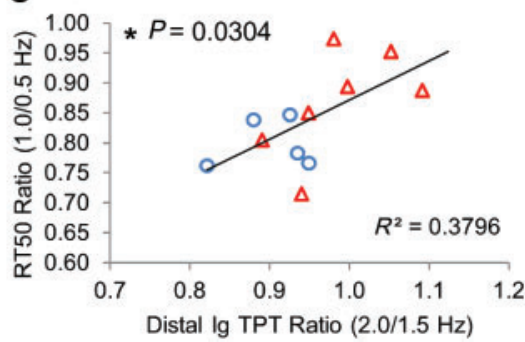

Figure 6 Relationship between tension by titin distal lg domain and cardiomyocytes FDAR in human hearts. Relative TPT of distal lg domain at $2.0 \mathrm{~Hz}$ was positively correlated with RT50 ratio (A) and RT90 ratio (B) at 1.0 over $0.5 \mathrm{~Hz}$ of cardiomyocytes. The significant correlations suggested the higher the TPT, the poorer the FDAR in myocytes. (C) RT50 ratio of cardiomyocytes was positively correlated with TPT ratio of distal Ig domain significantly. The poorer the FDAR in myocytes, the lesser reduction or even bigger increase the TPT at higher heart rate. Statistic was done by Pearson analysis, $* P<0.05$.

the underlying mechanism, we studied titin SMFS at increasing 'heart rates', and its relationship with FDAR response of the isolated myocytes from the same failing and non-failing human hearts. We showed that on average in the entire cohort, tested titin domains lowered their passive tension at a higher heart rate; and this characteristic in distal Ig domain alone was positively correlated with a greater FDAR in cardiomyocytes, which suggested a better ventricular filling upon a raised heart rate. The reduction of TPT at a higher heart rate was significantly blunted in failing hearts for the distal Ig domain alone, greater than that for PEVK-distal Ig domain. Together with the significant correlations found between the data from distal Ig domain alone and cardiomyocytes, it can be speculated that distal Ig domain is more vulnerable than PEVK domain to the impairment of TPT changes according to a raised heart rate. This would potentially support by the fact that $\mathrm{g}$ domain unfolds first during diastole, followed by PEVK domain. ${ }^{36,37}$ Our finding on TPT reduction seemed contradictory to the previous notion that faster stretching increased titin unfolding forces. $^{23,49,57-59}$ However, these studies differed from us by fully unfolding recombinant titin domains at large ranges of pulling speeds. Moreover, we included the refolding steps, which is unneglectable in assessing titin elastic energy. ${ }^{19,20,35}$

Additionally, we observed that the higher the TPT in distal Ig domain, the faster the cardiomyocytes relaxed, but the poorer the FDAR response. Although further definitive experiments are indispensable for a causal inference, these findings suggested a potential role of titin distal lg domain in regulating cardiac relaxation and kinetic reserve. Although the mean TPT was lowered at a higher heart rate in the entire cohort, this reduction in failing hearts was blunted to nearly unchanged, with some even reversed. Trends also existed that such diminished TPT reduction in distal Ig domain of failing hearts was associated with poorer FDAR. Both TPT and myocyte relaxation from failing hearts did not show significant differences from the non-failing hearts until comparing the changes upon a raised heart rate. Coincidently, HF patients commonly experience no symptom at rest but only during physical activities or stress. $^{5,21,22}$

When the heart rate increases, FDAR occurs along with a decrease in myofilament calcium sensitivity. ${ }^{11}$ This frequency-dependent myofilament calcium desensitization correlates with troponin I ( $\mathrm{Tnl}$ ) phosphorylation, which is a key regulator in myocardial function. ${ }^{7,60} \mathrm{Tnl}$ is phosphorylated by protein kinase A, protein kinase $C$ (PKC), and protein kinase $G$, which are well reported on altering TPT. ${ }^{15} \mathrm{PKC} \alpha$, the predominant isozyme in the heart, phosphorylates titin PEVK region, with one site close to distal Ig domain, increases TPT. ${ }^{48}$ At high-frequency stimulation on rat myocardium, the phosphorylation levels of PKC $\alpha$ decrease in controls but significantly increase in failing hearts. ${ }^{61}$ This study agrees with our data that mean TPT on the two tested domains decreases at higher heart rates but at a lesser extent in failing human hearts. Post-translational modification behind our findings merit further investigations.

Correlational studies employed in our study embraced the inherent human-to-human variability. This variability is typically absent in small rodent models, since these inbred models offer no genotype variability, and a very low phenotypical variability, and thus offer a too narrow range for meaningful correlation studies. The vast diversity in the human population, however, easily allows a broad-range of correlative outcomes to be investigated and compared. Our results provided key mechanical evidence from human hearts not only at a cellular level but also at a protein level to our further understanding of impaired myocardial relaxation in HF.

\subsection{Limitations of the study}

Firstly, the present study was hypothesis generating rather than definitive proof that titin kinetics contribute to impaired myocardial relaxation. Unlike animal models, loss/gain-of-function studies for determining causal mechanisms on human samples are highly limited. Secondly, same as all the other research using human myocardium, an appropriate control group was a challenging issue. The majority of previous research using live human end-stage failing heart tissue is done without the perfect non-failing control (a perfect non-failing control heart would be used for transplantation). However, although not completely exempt from cardiac-related medical history, donors from whom we obtained nonfailing hearts for this study were generally reported to have non-cardiacrelated causes of death. However, recent studies from our laboratory, using some of the same hearts as used for this study, showed clear functional phenotype differences between the failing human heart and the non-failing donor hearts used. ${ }^{62-65}$ Additionally, potential effects of the patients' and donors' medication history on cardiomyocytes and titin mechanics cannot be assessed, a known limitation that is unavoidable when working with human tissue. Moreover, in our model of unloaded intact cardiomyocytes, sarcomere lengths are shorter, and the ratelimiting process that regulate relaxation may differ from that in vivo. ${ }^{7}$ 
Although being an important candidate in counteracting myocyte from over-lengthening, little is known about the role of TPT in unloaded intact myocytes. ${ }^{66}$ Furthermore, with the overall physiological and functional mechanism elucidated in our present study, the molecular mechanism behind frequency-dependent changes of TPT requires further investigation, with studies into post-translational modification of titin the most likely venue. Lastly, the likelihood of specific protein picks up by AFM probe in our approach is very low, which can be improved for a better efficiency.

\section{Summary}

Here, we developed a novel force spectroscopy approach to detect passive tension by titin domains physiologically from failing and non-failing human hearts. The average tension on titin during cardiac cycle reduced upon an increased heart rate, while this reduction was significantly blunted in failing human hearts. These results were correlated with the relaxation kinetics of isolated human LV cardiomyocytes. Our findings suggested the importance of titin tension level and its dynamic changes in cardiomyocytes to speed up relaxation at higher heart rate, which was impaired in HF.

\section{Supplementary material}

Supplementary material is available at Cardiovascular Research online.

\section{Acknowledgements}

We thank Siegfried Labeit (University of Heidelberg, Germany) for the generous gift of antibody 184. We thank the Lifeline of Ohio Organ Procurement programme for their collaboration in obtaining donor hearts for research.

\section{Conflict of interest: none declared.}

\section{Funding}

This work was supported by NIH grants RO1HL113084 and RC1HL099538 (to P.M.L.J.), and T32NS779844 (to M.-P.C.).

\section{References}

1. Janssen PM. Myocardial contraction-relaxation coupling. Am J Physiol Heart Circ Physiol 2010;299:H1741-H1749.

2. Paulus WJ, Tschope C. A novel paradigm for heart failure with preserved ejection fraction: comorbidities drive myocardial dysfunction and remodeling through coronary microvascular endothelial inflammation. J Am Coll Cardiol 2013;62:263-271.

3. Janssen PML, Biesiadecki BJ, Ziolo MT, Davis JP. The need for speed: mice, men, and myocardial kinetic reserve. Circ Res 2016;119:418-421.

4. Rossman El, Petre RE, Chaudhary KW, Piacentino V 3rd, Janssen PM, Gaughan JP, Houser SR, Margulies KB. Abnormal frequency-dependent responses represent the pathophysiologic signature of contractile failure in human myocardium. J Mol Cell Cardiol 2004;36:33-42.

5. Wachter R, Schmidt-Schweda S, Westermann D, Post H, Edelmann F, Kasner M, Luers C, Steendijk P, Hasenfuss G, Tschope C, Pieske B. Blunted frequencydependent upregulation of cardiac output is related to impaired relaxation in diastolic heart failure. Eur Heart J 2009;30:3027-3036.

6. Varian KD, Biesiadecki BJ, Ziolo MT, Davis JP, Janssen PM. Staurosporine inhibits frequency-dependent myofilament desensitization in intact rabbit cardiac trabeculae. Biochem Res Int 2012;2012:1.

7. Janssen PM, Periasamy M. Determinants of frequency-dependent contraction and relaxation of mammalian myocardium. J Mol Cell Cardiol 2007;43:523-531.

8. Tskhovrebova L, Trinick J, Sleep JA, Simmons RM. Elasticity and unfolding of single molecules of the giant muscle protein titin. Nature 1997;387:308-312.
9. Mulieri LA, Hasenfuss G, Leavitt B, Allen PD, Alpert NR. Altered myocardial forcefrequency relation in human heart failure. Circulation 1992;85:1743-1750.

10. Janssen PM, Stull LB, Marban E. Myofilament properties comprise the rate-limiting step for cardiac relaxation at body temperature in the rat. Am J Physiol Heart Circ Physiol 2002;282:H499-H507.

11. Varian KD, Janssen PM. Frequency-dependent acceleration of relaxation involves decreased myofilament calcium sensitivity. Am J Physiol Heart Circ Physiol 2007;292: $\mathrm{H} 2212-\mathrm{H} 2219$.

12. Kassiri Z, Myers R, Kaprielian R, Banijamali HS, Backx PH. Rate-dependent changes of twitch force duration in rat cardiac trabeculae: a property of the contractile system. J Physiol (Lond) 2000;524(pt. 1):221-231.

13. Chung CS, Granzier HL. Contribution of titin and extracellular matrix to passive pressure and measurement of sarcomere length in the mouse left ventricle. J Mol Cell Cardiol 2011;50:731-739.

14. Hamdani N, Paulus WJ. Myocardial titin and collagen in cardiac diastolic dysfunction: partners in crime. Circulation 2013;128:5-8.

15. Linke WA, Hamdani N. Gigantic business: titin properties and function through thick and thin. Circ Res 2014;114:1052-1068.

16. Helmes M, Lim CC, Liao R, Bharti A, Cui L, Sawyer DB. Titin determines the FrankStarling relation in early diastole. J Gen Physiol 2003;121:97-110.

17. Chen MP, Li SN, Lam WW, Ho YC, Ha SY, Chan GC, Cheung YF. Left ventricular torsional mechanics and myocardial iron load in beta-thalassaemia major: a potential role of titin degradation. BMC Cardiovasc Disord 2014;14:49.

18. Castro-Ferreira R, Fontes-Carvalho R, Falcão-Pires I, Leite-Moreira AF. The role of titin in the modulation of cardiac function and its pathophysiological implications. Arq Bras Cardiol 2011;96:332-339.

19. Kötter S, Gout L, Von Frieling-Salewsky M, Müller AE, Helling S, Marcus K, Dos Remedios C, Linke WA, Krüger M. Differential changes in titin domain phosphorylation increase myofilament stiffness in failing human hearts. Cardiovasc Res 2013;99: 648-656.

20. Zile MR, Baicu CF, Ikonomidis JS, Stroud RE, Nietert PJ, Bradshaw AD, Slater R, Palmer BM, Van Buren P, Meyer M, Redfield MM, Bull DA, Granzier HL, LeWinter MM. Myocardial stiffness in patients with heart failure and a preserved ejection fraction: contributions of collagen and titin. Circulation 2015;131:1247-1259.

21. Phan TT, Abozguia K, Nallur Shivu G, Mahadevan G, Ahmed I, Williams L, Dwivedi G, Patel K, Steendijk P, Ashrafian H, Henning A, Frenneaux M. Heart failure with preserved ejection fraction is characterized by dynamic impairment of active relaxation and contraction of the left ventricle on exercise and associated with myocardial energy deficiency. J Am Coll Cardiol 2009;54:402-409.

22. Chattopadhyay S, Alamgir MF, Nikitin NP, Rigby AS, Clark AL, Cleland JG. Lack of diastolic reserve in patients with heart failure and normal ejection fraction. Circ Heart Fail 2010;3:35-43.

23. Linke WA, Grutzner A. Pulling single molecules of titin by AFM-recent advances and physiological implications. Pflugers Arch 2008;456:101-115.

24. Wang SM, Greaser ML. Immunocytochemical studies using a monoclonal antibody to bovine cardiac titin on intact and extracted myofibrils. J Muscle Res Cell Motil 1985;6: 293-312.

25. Furst DO, Osborn M, Nave R, Weber K. The organization of titin filaments in the half-sarcomere revealed by monoclonal antibodies in immunoelectron microscopy: a map of ten nonrepetitive epitopes starting at the $Z$ line extends close to the $M$ line. J Cell Biol 1988;106:1563-1572.

26. Chung CS, Hutchinson KR, Methawasin M, Saripalli C, Smith JE, Hidalgo CG, Luo X, Labeit S, Guo C, Granzier HL. Shortening of the elastic tandem immunoglobulin segment of titin leads to diastolic dysfunction. Circulation 2013;128:19-28.

27. Martonfalvi Z, Bianco P, Linari M, Caremani M, Nagy A, Lombardi V, Kellermayer M. Low-force transitions in single titin molecules reflect a memory of contractile history. J Cell Sci 2014;127:858-870.

28. Trombitas K, Greaser M, French G, Granzier H. PEVK extension of human soleus muscle titin revealed by immunolabeling with the anti-titin antibody 9D10. J Struct Biol 1998;122:188-196.

29. Trombitas K, Redkar A, Centner T, Wu Y, Labeit S, Granzier H. Extensibility of isoforms of cardiac titin: variation in contour length of molecular subsegments provides a basis for cellular passive stiffness diversity. Biophys J 2000;79:3226-3234.

30. Granzier HL, Hutchinson KR, Tonino P, Methawasin M, Li FW, Slater RE, Bull MM, Saripalli C, Pappas CT, Gregorio CC, Smith JE. Deleting titin's I-band/A-band junction reveals critical roles for titin in biomechanical sensing and cardiac function. Proc Natl Acad Sci U S A 2014;111:14589-14594.

31. Linke WA, Ivemeyer M, Mundel P, Stockmeier MR, Kolmerer B. Nature of PEVK-titin elasticity in skeletal muscle. Proc Natl Acad Sci U S A 1998;95:8052-8057.

32. Granzier HL, Radke MH, Peng J, Westermann D, Nelson OL, Rost K, King NM, Yu Q, Tschope C, McNabb M, Larson DF, Labeit S, Gotthardt M. Truncation of titin's elastic PEVK region leads to cardiomyopathy with diastolic dysfunction. Circ Res 2009;105:557-564.

33. Alegre-Cebollada J, Kosuri P, Giganti D, Eckels E, Rivas-Pardo JA, Hamdani N, Warren CM, Solaro RJ, Linke WA, Fernández JM. S-glutathionylation of cryptic cysteines enhances titin elasticity by blocking protein folding. Cell 2014;156:1235-1246.

34. Grutzner A, Garcia-Manyes S, Kotter S, Badilla CL, Fernandez JM, Linke WA. Modulation of titin-based stiffness by disulfide bonding in the cardiac titin N2-B unique sequence. Biophys / 2009;97:825-834. 
35. Helmes M, Trombitas K, Centner T, Kellermayer M, Labeit S, Linke WA, Granzier H. Mechanically driven contour-length adjustment in rat cardiac titin's unique N2B sequence: titin is an adjustable spring. Circ Res 1999;84:1339-1352.

36. Linke WA, Rudy DE, Centner T, Gautel M, Witt C, Labeit S, Gregorio CC. I-band titin in cardiac muscle is a three-element molecular spring and is critical for maintaining thin filament structure. J Cell Biol 1999;146:631-644.

37. Wang K, Forbes JG, Jin AJ. Single molecule measurements of titin elasticity. Prog Biophys Mol Biol 2001;77:1-44.

38. Backmann N, Kappeler N, Braun T, Huber F, Lang HP, Gerber C, Lim RY. Sensing surface PEGylation with microcantilevers. Beilstein J Nanotechnol 2010;1:3-13.

39. Wu X, Sun Z, Foskett A, Trzeciakowski JP, Meininger GA, Muthuchamy M. Cardiomyocyte contractile status is associated with differences in fibronectin and integrin interactions. Am J Physiol Heart Circ Physiol 2010;298:H2071-H2081.

40. Li M, Liu L, Xi N, Wang Y, Xiao X, Zhang W. Imaging and measuring the biophysical properties of $F_{c}$ gamma receptors on single macrophages using atomic force microscopy. Biochem Biophys Res Commun 2013;438:709-714.

41. Sulchek TA, Friddle RW, Langry K, Lau EY, Albrecht H, Ratto TV, DeNardo SJ, Colvin ME, Noy A. Dynamic force spectroscopy of parallel individual Mucin1antibody bonds. Proc Natl Acad Sci U S A 2005;102:16638-16643.

42. Wiggers C], Modern Aspects of the Circulation in Health and Disease. Philadelphia/New York: Lea and Febiger; 1915.

43. Anderson BR, Granzier HL. Titin-based tension in the cardiac sarcomere: molecular origin and physiological adaptations. Prog Biophys Mol Biol 2012;110:204-217.

44. Rief M, Gautel M, Oesterhelt F, Fernandez JM, Gaub HE. Reversible unfolding of individual titin immunoglobulin domains by AFM. Science 1997;276:1109-1112.

45. Kellermayer MS, Smith SB, Granzier HL, Bustamante C. Folding-unfolding transitions in single titin molecules characterized with laser tweezers. Science 1997;276:1112-1116.

46. Anderson BR, Bogomolovas J, Labeit S, Granzier H. Single molecule force spectroscopy on titin implicates immunoglobulin domain stability as a cardiac disease mechanism. J Biol Chem 2013;288:5303-5315.

47. DuVall MM, Gifford JL, Amrein M, Herzog W. Altered mechanical properties of titin immunoglobulin domain 27 in the presence of calcium. Eur Biophys J 2013;42:301-307.

48. Hidalgo C, Hudson B, Bogomolovas J, Zhu Y, Anderson B, Greaser M, Labeit S, Granzier H. PKC phosphorylation of titin's PEVK element: a novel and conserved pathway for modulating myocardial stiffness. Circ Res 2009;105:631-638, 17 p following 638.

49. Zhu Y, Bogomolovas J, Labeit S, Granzier H. Single molecule force spectroscopy of the cardiac titin N2B element: effects of the molecular chaperone alphaB-crystallin with disease-causing mutations. J Biol Chem 2009;284:13914-13923.

50. Kruger M, Kotter S, Grutzner A, Lang P, Andresen C, Redfield MM, Butt E, dos Remedios CG, Linke WA. Protein kinase G modulates human myocardial passive stiffness by phosphorylation of the titin springs. Circ Res 2009;104:87-94.

51. Kellermayer MS, Bustamante C, Granzier HL. Mechanics and structure of titin oligomers explored with atomic force microscopy. Biochim Biophys Acta 2003;1604: 105-114.
52. Trombitas K, Freiburg A, Centner T, Labeit S, Granzier H. Molecular dissection of N2B cardiac titin's extensibility. Biophys J 1999;77:3189-3196.

53. Linke WA, Granzier H. A spring tale: new facts on titin elasticity. Biophys J 1998;75: 2613-2614.

54. Minajeva A, Kulke M, Fernandez JM, Linke WA. Unfolding of titin domains explains the viscoelastic behavior of skeletal myofibrils. Biophys / 2001;80:1442-1451.

55. Komamura K. Similarities and differences between the pathogenesis and pathophysiology of diastolic and systolic heart failure. Cardiol Res Pract 2013;2013: 824135.

56. Francis GS, Thenappan T. The end is near... do we know everything about cardiopulmonary exercise testing in HF patients? J Am Coll Cardiol 2016;67:790-792

57. Watanabe K, Muhle-Goll C, Kellermayer MS, Labeit S, Granzier H. Different molecular mechanics displayed by titin's constitutively and differentially expressed tandem Ig segments. J Struct Biol 2002;137:248-258.

58. Best RB, Fowler SB, Herrera JL, Steward A, Paci E, Clarke J. Mechanical unfolding of a titin Ig domain: structure of transition state revealed by combining atomic force microscopy, protein engineering and molecular dynamics simulations. J Mol Biol 2003; 330:867-877.

59. Bianco P, Mártonfalvi Z, Naftz K, Köszegi D, Kellermayer M. Titin domains progressively unfolded by force are homogenously distributed along the molecule. Biophys J 2015; 109:340-345.

60. Layland J, Solaro RJ, Shah AM. Regulation of cardiac contractile function by troponin I phosphorylation. Cardiovasc Res 2005;66:12-21.

61. Lamberts RR, Hamdani N, Soekhoe TW, Boontje NM, Zaremba R, Walker LA, de Tombe PP, van der Velden J, Stienen GJ. Frequency-dependent myofilament Ca2+ desensitization in failing rat myocardium. J Physiol (Lond) 2007;582:695-709.

62. Chung JH, Canan BD, Whitson BA, Kilic A, Janssen PML. Force-frequency relationship and early relaxation kinetics are preserved upon sarcoplasmic blockade in human myocardium. Physiol Rep 2018;6:e13898.

63. Chung JH, Martin BL, Canan BD, Elnakish MT, Milani-Nejad N, Saad NS, Repas SJ, Schultz JEJ, Murray JD, Slabaugh JL, Gearinger RL, Conkle J, Karaze T, Rastogi N, Chen MP, Crecelius W, Peczkowski KK, Ziolo MT, Fedorov VV, Kilic A, Whitson BA, Higgins RSD, Smith SA, Mohler PJ, Binkley PF, Janssen PML. Etiology-dependent impairment of relaxation kinetics in right ventricular end-stage failing human myocardium. J Mol Cell Cardiol 2018;121:81-93.

64. Janssen PML, Canan BD, Kilic A, Whitson BA, Baker AJ. Human myocardium has a robust alpha1A-subtype adrenergic receptor inotropic response. I Cardiovasc Pharmacol 2018;72:136-142.

65. Elnakish MT, Canan BD, Kilic A, Mohler PJ, Janssen PM. Effects of zacopride, a moderate IK1 channel agonist, on triggered arrhythmia and contractility in human ventricular myocardium. Pharmacol Res 2017;115:309-318.

66. King NM, Methawasin M, Nedrud J, Harrell N, Chung CS, Helmes M, Granzier H. Mouse intact cardiac myocyte mechanics: cross-bridge and titin-based stress in unactivated cells. J Gen Physiol 2011;137:81-91. 